\title{
COMMUNICATION
}

\section{Structural Characterization of an Unprecedented Lectin-like Antitumoral anti-MUC1 Antibody}

Received 00th January 20xx, Accepted 00th January 20xx

DOI: $10.1039 / \times 0 \times x 00000 x$

Javier Macías-León, ${ }^{a}$ Iris A. Bermejo, ${ }^{\text {b }}$ Alicia Asín, ${ }^{\text {b }}$ Ana García-García, ${ }^{a}$ Ismael Compañón, ${ }^{b}$ Ester Jiménez-Moreno, ${ }^{b}$ Helena Coelho, ${ }^{c}$ Vincenzo Mangini, ${ }^{d}$ Inês S. Albuquerque, ${ }^{e}$ Filipa Marcelo, ${ }^{c}$ Juan L. Asensio, ${ }^{f}$ Gonçalo J. L. Bernardes, ${ }^{e, g}$ Hiren J. Joshi, ${ }^{h}$ Roberto Fiammengo, ${ }^{d}$ Ola Blixt, ${ }^{\text {h }}$ Ramón Hurtado-Guerrero, ${ }^{*, a, h, i, j}$

Francisco

Corzana*,b

The molecular basis of antibody 5E5, which recognizes the entire GalNAc unit as a primary epitope is disclosed. The antibody's contacts with the peptide are limited to mostly two residues, allowing it to show some degree of promiscuity. These findings open the door to the chemical design of peptide-mimetics for developing efficient anti-cancer vaccines and diagnostic tools.

Mucins are heavily glycosylated proteins that play a key role in several biological processes. MUC1 is one of the most studied mucins. ${ }^{1}$ Although MUC1 exhibits complex $O$-glycans in healthy cells, in tumour tissues it is decorated with short carbohydrates. Consequently, different antigens that are masked in healthy cells, such as the Tn and STn antigens ${ }^{2,3}$ (GalNAc- $\alpha-1-O-T h r / S e r$ and Neu5Ac- $\alpha-(2-6)$-GalNAc- $\alpha-1-O-T h r / S e r$, respectively), are exposed in cancer cells and can trigger an immune response. These antigens are broadly expressed in many types of cancer and are well-accepted hallmarks of this disease. ${ }^{4,5}$ Despite the vast amount of clinical and immunological data available on antibodies that recognize aberrantly glycosylated MUC1, the molecular details by which these antibodies recognize their targets are scarce. ${ }^{6}$ This structural knowledge is fundamental to the development of new anti-MUC1 antibodies with improved specificities to advance cancer therapy. ${ }^{7}$ Also, to design new MUC1-inspired glycopeptides with enhanced binding strength and specificity for naturally occurring anti-MUC1 antibodies present in cancer patients to improve early cancer detection. ${ }^{8}$

a. Institute of Biocomputation and Physics of Complex Systems (BIFI), University of Zaragoza, Mariano Esquillor s/n, Campus Rio Ebro, Edificio I+D, Zaragoza, Spain.

${ }^{b .}$ Department of Chemistry, University of La Rioja, 26006, Logroño, Spain.

UCIBIO, REQUIMTE, Departamento de Química, Faculdade de Ciências e Tecnologia, Universidade NOVA de Lisboa, Portugal.

d.Center for Biomolecular Nanotechnologies@UniLe, Istituto Italiano di Tecnologia (IIT), 73010 Arnesano, Lecce, Italy.

e. Instituto de Medicina Molecular, Faculdade de Medicina, Universidade de Lisboa, Lisboa, Portugal.

f. Instituto de Química Organica General, IQOG-CSIC, 28006 Madrid, Spain.

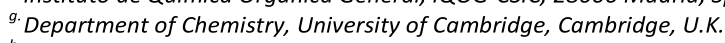

h. Copenhagen Center for Glycomics, Department of Cellular and Molecular Medicine, University of Copenhagen, Copenhagen, Denmark.

Laboratorio de Microscopías Avanzada (LMA), University of Zaragoza.Campus Rio Ebro, Edificio 1 $+D$, Zaragoza, Spain.

Fundación ARAID, Zaragoza, Spain

Electronic Supplementary Information (ESI) available: [details of any supplementary information available should be included here]. See DOI: $10.1039 / x 0 x x 00000 x$
From a molecular recognition perspective, although most of anti-MUC1 monoclonal antibodies (mAb) recognize the peptide sequence DTRP, recent studies indicate that some bind to GSTAP or GVTS motifs. ${ }^{9-11}$ The unusual and incomplete glycosylation of MUC1 modulates the binding properties of these antibodies. ${ }^{7}$ In general, binding is enhanced by glycosylation of the PDTR epitope. In this respect, the X-ray structure of the complex between antibody AR20.5 and glycopeptide APDT*RP (in which $\mathrm{T}^{*}=\mathrm{Tn}-\mathrm{Thr}=$ GalNAc- $\left.\alpha-1-O-T h r\right)$ reveals that the contacts between the sugar and the antibody are negligible. ${ }^{6}$ However, the presence of the sugar improves glycopeptide binding because it stabilizes the conformation of the peptide that is recognized by the antibody. Similarly, SM3, recognizes mainly the peptide sequence and the sugar adopts a conformation that provides several additional stabilizing interactions with the antibody. ${ }^{12}$

To date, two high-resolution X-ray structure of a complex between an antibody and a glycopeptide, in which the carbohydrate is part of the epitope have been reported. ${ }^{13,14}$ However, only one of these antibodies (named SN-101) refers to anti-MUC1 antibodies. SN-101 was designed specifically for this purpose following a smart strategy developed by Nishimura's group. ${ }^{14} Y e t$, in this case, the GalNAc is not intimately recognized by the $\mathrm{SN}-101$, and just GalNAc $\mathrm{OH} 6$ and $\mathrm{OH} 4$ are interacting with the antibody.

The anti-MUC1 antibody 5E5 is an ideal candidate for detailed structural investigations since it recognizes mainly the sequence GSTAP and requires a GaINAc attached to the threonine residue. ${ }^{11}$ Besides, it has been successfully used to develop chimeric antigen receptor T cells (CAR-T cells). ${ }^{15}$ Notably, recent studies propose that 5E5 could recognize tumour cell lines that do not express MUC1-like glycopeptides, which suggests a certain degree of promiscuity. ${ }^{15,16}$ Initially, scFv-5E5 was cloned, expressed and purified to homogeneity to enable functional and structural characterization (ESI and Fig. S1). We evaluated then substrate specificity by isothermal titration calorimetry (ITC) measurements (Fig. 1A, S2-S3 and Table S1). According to these experiments, scFv-5E5 displays the highest affinity against glycopeptides $\mathbf{1}$ and the longer variant 1', whereas it does not bind to unglycosylated derivative. We could not determine any binding of the antibody against GalNAc- $\alpha-1-O-M e$, probably due to the very low affinity, as previously reported. ${ }^{11}$ Although the presence of two Tn antigens in the peptide (glycopeptide 3) modestly affects binding (Fig. 1), attachment of a sialic acid 
moiety to the hydroxymethyl group of GalNAc (STn-containing glycopeptide 4) results in considerably weaker binding. This result is corroborated by the low signal-to-noise ratio observed in the recorded ITC profile and agrees with the preferences reported earlier. ${ }^{10}$ A similar result, in terms of affinity, was obtained when the glycopeptide $\mathbf{2}$ was tested. This clearly suggests that the antibody recognizes other regions of the tandem-repeat sequence of MUC1 and possibly other proteins as described before. ${ }^{16,17}$

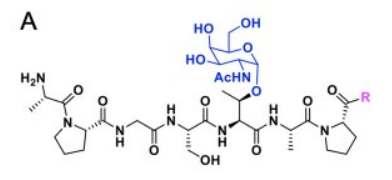

APGST*AP $\left(\mathrm{R}=\mathrm{NH}_{2}, 1\right)$ APGST*APPAHGV ( $\left(R=\right.$ peptide PAHGV, $\left.1^{\prime}\right)$

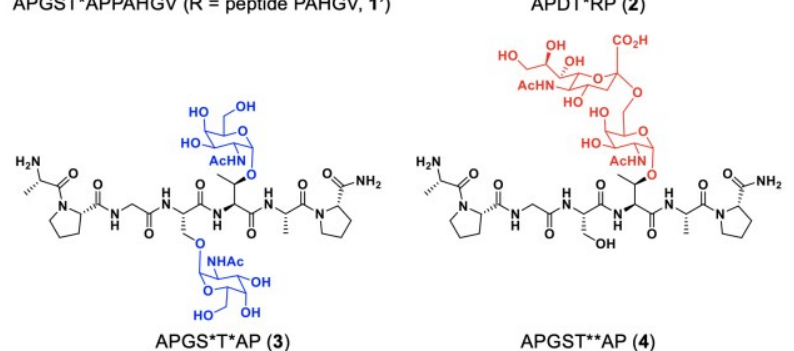

B

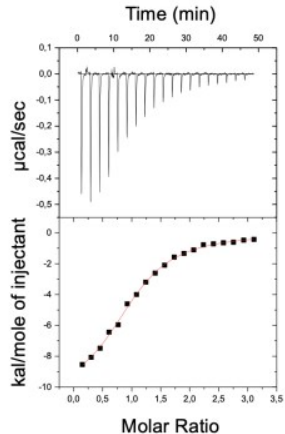

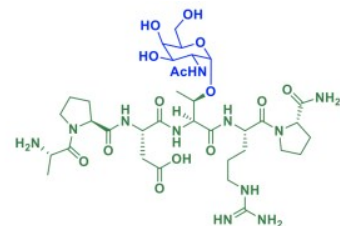

APDT*RP (2)

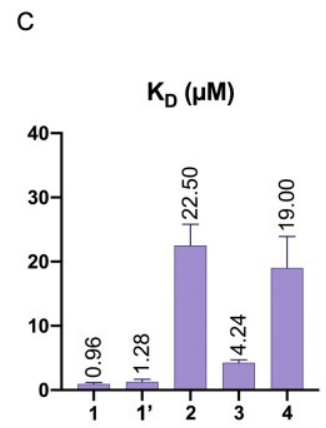

Fig. 1. (A) Glycopeptides synthesized and studied in this work. (B) ITC profiles for glycopeptide 3 titration with $20 \mu \mathrm{M}$ scFv-5E5 solution at 25 ${ }^{\circ} \mathrm{C}$ and $\mathrm{pH}=7.5$. (C) $\mathrm{K}_{\mathrm{D}}$ of glycopeptides $1, \mathbf{1}^{\prime}$ and 2-4 with scFv-5E5 determined by ITC. (see also Table S1 and Fig. S2-S3).

Further, we successfully obtained crystals of scFv-5E5 in a complex with compound 1 (Fig. 2, pdb ID: 6TNP). The resulting crystals allowed us to solve the structure at a resolution of $3.00 \AA$ and interpret the density map (Table S2 and Fig. S5). The glycopeptide lies within a surface groove formed by the light (L) and heavy $(\mathrm{H})$ chains, and in particular is recognized by residues of the loops $\beta 3^{\mathrm{H}}-\beta 4^{\mathrm{H}}, \beta 9^{\mathrm{H}}-\beta 10^{\mathrm{H}}, \beta 8^{\mathrm{L}}-\beta 9^{\mathrm{L}}$ and $\beta 4^{\mathrm{H}}$ (Fig. 2). The GalNAc moiety is the most recognized part of the glycopeptide. Indeed, with exception of the $\mathrm{OH} 6$, all its hydroxyl groups are engaged in hydrogen bonds. In detail, hydroxyl group $\mathrm{OH} 3$ interacts with the $\mathrm{NH}$ group of $\mathrm{Ala}_{3} 3^{\mathrm{H}}$ and $\mathrm{OH} 4$ with the side chains of $\mathrm{His}_{32} \mathrm{H}^{\mathrm{H}}$ and Ser99 ${ }^{\mathrm{H}}$. Equally, the endocyclic oxygen of the sugar is engaged in hydrogen bonding with Ser9 ${ }^{\mathrm{H}}$. The carbonyl group of GalNAc is involved in two hydrogen bonds with the side chain of $\mathrm{His} 35^{\mathrm{H}}$ and $\mathrm{Thr} 100^{\mathrm{H}}$ and the methyl group is engaged in a $\mathrm{CH} / \pi$ stacking interaction with $\mathrm{His} 50^{\mathrm{H}}$, which provides the impetus for the observed selectivity of 5E5 for GalNAc-containing antigens. The $\mathrm{OH} 6$ of the sugar is fully solvent-exposed, which explains why this antibody can also recognize STn-containing glycopeptides. ${ }^{10}$ The glycosidic linkage adopts the typical 'eclipsed' conformation, ${ }^{18,19}$ with $\phi_{2 / 2} \psi_{c}$ values of $68.1^{\circ} / 151.0^{\circ}$. In contrast to the carbohydrateg/the preptide moiety forms only one hydrogen bond with the protein. This stabilizing interaction involves the $-\mathrm{CONH}_{2}$ group in the $C$ terminal region of the glycopeptide and the carbonyl group of Tyr98. The $\mathrm{N}$-terminal region (APGS) is completely exposed to the solvent and does not establish any contact with the antibody. The saturation-transfer difference (STD)-NMR spectrum obtained for $\mathbf{1}$ in presence of scFv-5E5 agrees with this epitope deduced from the $\mathrm{X}$-ray structure (Fig. S6). This result is in striking contrast to that found for SN-101 antibody, ${ }^{14}$ in which a significant hydrogen bonding network is stablished between the antibody and the peptide. This feature presumably prevents its ability to recognize other peptide fragments. Notably, as in the case of SM3 $\mathrm{mAb},{ }^{12}$ the glycosylated Thr residue of the peptide adopts a helix-like conformation in the bound state. This conformer could be favoured by an intramolecular hydrogen bond between the $\mathrm{NH}$ of the contiguous Ala (Ala17) and the hydroxyl group of Ser15 (Fig. 2, dotted orange line), which in turn provides the proper shape complementarity with the antibody.

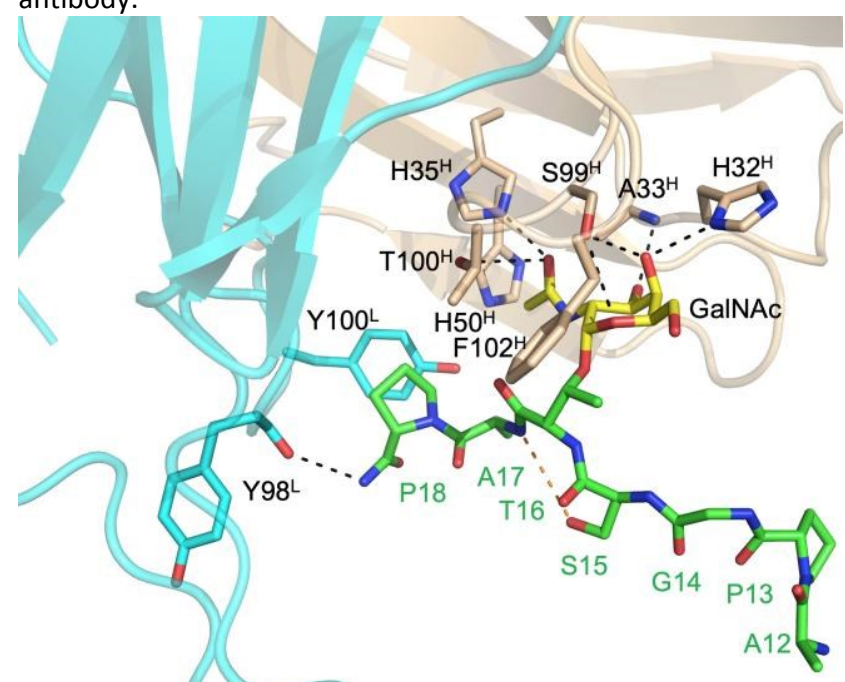

Fig. 2. X-ray structure of glycopeptide 1 bound to scFv-5E5, showing the hydrogen bond interactions. PDB ID: 6TNP.

Selectivity towards this peptide sequence (APGSTAP) is conferred by two $\mathrm{CH}-\pi$ stacking interactions that involve the methyl group of threonine and the five-membered ring of the $C$ terminal proline with the aromatic moieties of $\mathrm{Phe} 102^{\mathrm{H}}$ and Tyr $100^{L}$, respectively. The importance of these two aromatic residues and those of $\mathrm{His} 32^{\mathrm{H}}$ and $\mathrm{His} 35^{\mathrm{H}}$ for the binding was confirmed by Ala substitutions by using site-directed mutagenesis (Table S1). Binding of mutants $\mathrm{F} 102^{\mathrm{H}} \mathrm{A}, \mathrm{H} 32^{\mathrm{H}} \mathrm{A}^{\mathrm{H}}$ $\mathrm{H} 35^{\mathrm{H}} \mathrm{A}$ and $\mathrm{Y} 100^{\mathrm{L}} \mathrm{A}$ towards peptide $\mathbf{1}$ was severely impaired. Indeed, we could not detect clear binding for the first mutant, and a $\sim 1330$ - and $~ 790$-fold decrease in affinity was observed for $\mathrm{H} 32^{\mathrm{H}} \mathrm{A}^{\mathrm{H}}-\mathrm{H} 35^{\mathrm{H}} \mathrm{A}$ and $\mathrm{Y} 100^{\mathrm{L}} \mathrm{A}$ mutants, respectively. A similar result was obtained when the $C$-terminal Pro of glycopeptide 1 was replaced by an Ala residue ( $\sim 00$-fold decrease in binding, Table S1, Fig. S7-S8). We performed then a series of microarray experiments with synthetic mutations and glycoform substitutions. Glycopeptides obtained from solid phase peptide synthesis (SPPS) and microarray display were performed as previously described. ${ }^{20,21}$ Figure $3 \mathrm{~A}$ shows that Ala mutation throughout a MUC1-like glycopeptide sequence completely abrogates $5 \mathrm{E} 5$ binding only when $\mathrm{T}^{*}$ and Pro are mutated in the 
GST*AP sequence, and reproduce previous work that used a random mutation library including Ala mutations at the GST*AP sequence. ${ }^{4}$ These results support that the minimal epitope is $T^{*} A P$. Extended amino acid $X$ substitutions at Ala position, further establish the true 5E5 epitope as - $T^{*}-\mathrm{X}$-P- (Figure 3B), which complements the crystal analysis and previous data. ${ }^{4}$ Chemical synthesis also allowed us to conclude that extended glycoforms, such as cores Core-2 (C2), Core-3 (C3) and Core-4 (C4), were not tolerated by the antibody (Figure $3 \mathrm{C}$ ), which fits with hydrogen bonding requirements above commented. Thus, 5 E5 mAb probably interacts selectively with glycopeptides that comprise the $-T^{*}-X-P$ - motif, which is present in the MUC1 tandem-repeat sequence but also in many other proteins.

A $V_{1} \mathrm{~S}_{2} \mathrm{AP}_{3} \mathrm{D}_{4} \mathrm{~T}_{5} \mathrm{R}_{8} \mathrm{P}_{7} \mathrm{AP}_{8} \mathrm{G}_{9} \mathrm{~S}_{10} \mathrm{~T}^{*}{ }_{11} \mathrm{AP}_{12} \mathrm{P}_{13} \mathrm{AH}_{14} \mathrm{G}_{15} \quad \mathrm{~B}$ VTSAPDTRPAPGST*XPPAHG

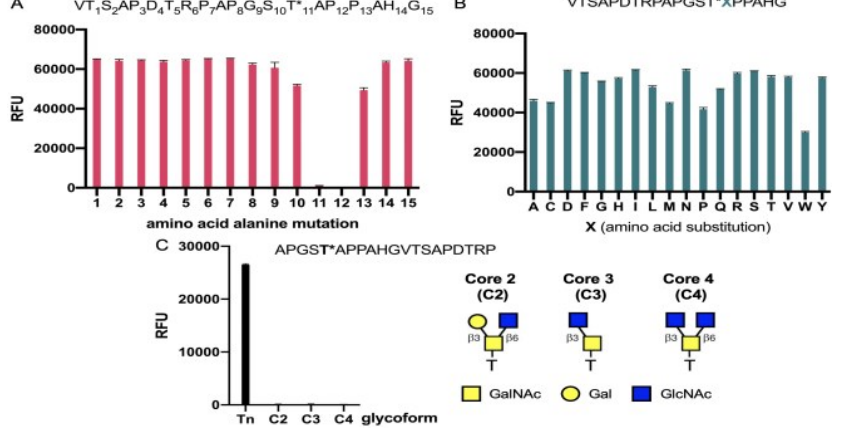

Fig. 3. (A) Microarray data for an Ala mutation walk through the entire 20-mer MUC1-Tn glycopeptide. (B) Microarray data for the T-*-X-P mutations in the GST*AP region. (C) Microarray data for the additional glycoform isomers $\mathbf{C 2}, \mathbf{C} 3$ and $\mathbf{C 4}$.

Putative 3D structures were then generated for the complexes formed between ScFv-5E5 and glycopeptides $\mathbf{1}, \mathbf{1}^{\prime}$ and $\mathbf{2 - 4}$, using the coordinates of the crystal structure of scFv-5E5/1. $0.5 \mu \mathrm{s}$ Molecular Dynamics (MD) simulations were run for each complex to produce fully equilibrated structures in water (Fig. 4 and S9-S19). It is important to note that in all simulated complexes the hydrogen bonds between GalNAc and the antibody observed in the X-ray structure are preserved (Fig. S18). With exception of complexes with derivatives $\mathbf{2}$ and $\mathbf{3}$, the $\mathrm{N}$-terminal region of the peptide was rather flexible, owing to the lack of stabilizing contacts between the peptide and the antibody. Complex scFv-5E5/1 was stable through the complete simulation and both the hydrogen bonds and the $\mathrm{CH}-\pi$ stacking interactions present in the $\mathrm{X}$-ray structure were highly populated. According to MD simulations, Pro19 and Ala20 of glycopeptide 1' (APGSTAPP ${ }_{19} \mathbf{A}_{20} \mathrm{HG}$ ) are engaged in $\mathrm{CH} / \pi$ interactions with Tyr $38^{L}$ and $T y r 98^{L}$, respectively, which agrees with reported data ${ }^{4}$ (Fig. S10). In this case, we could not determine the epitope mapping by STD-NMR due to the overlapping of key signals (Fig. S6). Presumably, the shorter peptide backbone of $\mathbf{1}$ related to $\mathbf{1}^{\prime}$ and the subsequent reduction in the entropic penalty associated with the binding process could compensate for these additional stabilizing interactions displayed by $\mathbf{1}^{\prime}$, as indicated by the $\mathrm{K}_{\mathrm{D}}$ values determined by ITC assays. The additional glycosylation of derivative 1 with GaINAc, which renders diglycopeptide 3, has two significant consequences in the bound state: (a) it rigidifies the $\mathrm{N}$-terminal moiety of the peptide; and (b) the Ser residue is forced to adopt a helix-like conformation, which is low populated in solution. ${ }^{22}$ The entropy penalty associated to binding, as confirmed by the ITC experiment (Table S1), could be counterbalanced by extra contacts between the GalNAc linked to Ser and 5E5, which explains the modest decrease of binding for this diglycopeptide. In case of complex ScFv-5E5/4, the MD simulations indicate that the Neu5Acl: 1anit39impairs63 the recognition of the peptide fragment. After 120 ns, the $\mathrm{CH}-\pi$ stacking interaction between the $C$-terminal Pro and Tyr $100^{L}$ is significantly attenuated due to the increasing distance between these residues (Fig. S19). This feature may explain why glycopeptide 4 binds worse than derivative 1 to the antibody.

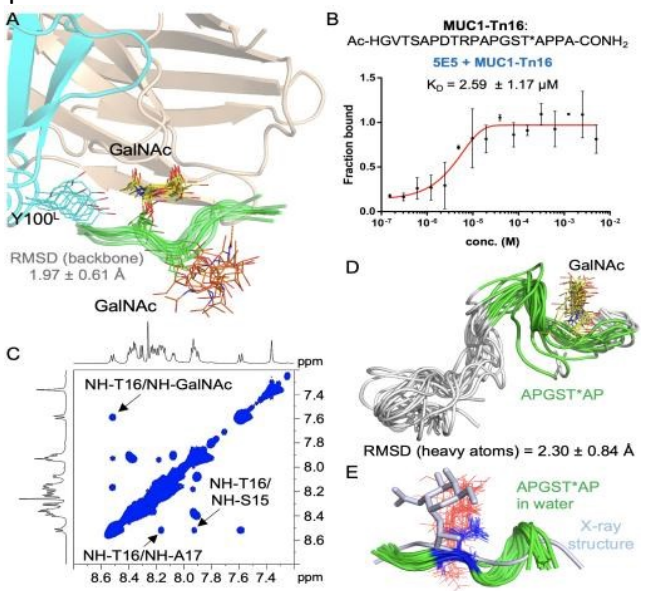

Fig. 4. (A) Overlay of 10 frames of scFv-5E5/3 complex sampled from 0.5 $\mu$ S MD simulations. RMSD value ( \pm S.D.) of the peptide backbone is shown. (B) MST binding curve used to determine $K_{D}$ for the complete 5E5 with glycopeptide MUC1-Tn16. Error represents SD between two separate experiments; $K_{D}$ values $\pm S D$ are shown. (C) Section of the 300 ms 2D-NOESY spectrum (600 MHz) in $\mathrm{H}_{2} \mathrm{O} / \mathrm{D}_{2} \mathrm{O}(9: 1)$ at $275 \mathrm{~K}$ and $\mathrm{pH} 5.5$ for MUC1-Tn16. (D) Structural ensembles derived from 200 ns experiment-guided MD simulations for MUC1-Tn16 in water. The RMSD value ( \pm S.D.) of heavy atoms is shown. (E) Superposition of the APGST*AP fragment of MUC1-Tn16 conformers found in solution with the conformation of glycopeptide 1 obtained from the $\mathrm{X}$-ray structure of scFv-5E5/1.

In the scFv-5E5/2 complex, the Asp residue is forced to adopt a helix-like conformation to favour the shape complementarity in the binding site. Taking into account the extended disposition adopted by this glycopeptide in solution, ${ }^{7,12,23}$ this conformational change in the 5E5-bound state can explain the entropy cost associated to the binding and, consequently, the lower affinity of scFv-5E5 towards glycopeptide 2. We also performed MD simulations of glycopeptide APGSS*AP (1-Ser, Fig. S20), in which the Thr residue was replaced by Ser, bound to scFv-5E5. The $\mathrm{CH}-\pi$ stacking interactions that involve $\mathrm{F} 102^{\mathrm{H}}$ and $\mathrm{Y} 100^{\mathrm{L}}$ are harmed at the beginning of the simulation and only the stabilizing interactions between GaINAc and the antibody are preserved. This outcome implies that $5 \mathrm{E} 5$ would only recognize proteins containing the motif $-\mathrm{T}^{*}-\mathrm{X}-\mathrm{P}$. This also agrees with our previous data where TS(GaINAc)AP-motif within MUC1 tandem repeat did not serve as a ligand for $5 \mathrm{E} 5 .^{20}$ We performed then a conformational analysis of glycopeptide MUC1-Tn16 (Fig. 4B). MUC1-Tn16 is recognized by scFv-5E5 and complete antibody $5 E 5$ with $K_{D}$ values of 3.42 and $2.59 \mu \mathrm{M}$, respectively (Fig. 4B and S4). The analysis of the NMR spectra for this compound (Table S3, Fig. 4C, S21 and S22), allowed us to obtain 68 NMR-derived distances (Table S4) that were used as restraints in experiment-guided MD simulations (ESI). The peptide backbone is rather flexible in water, particularly for those amino acids at distal positions from the glycosylation point and the GalNAc moiety forces the underlying threonine to adopt and extended conformation, in line with previous studies (Fig. $4 \mathrm{D}, 4 \mathrm{E}, \mathrm{S} 23)^{23,24}$ Thus, as the SM3 antibody, $5 \mathrm{E} 5$ selects a 
conformer poorly populated in solution. This result contrasts to that found for SN-101, for which the structure of the glycopeptide in bound state is identical to its solution NMRderived structure. In conclusion, we have solved the crystal structure of the antitumoral antibody 5E5 in complex with its natural epitope. Our findings establish that the main driving force of $5 E 5 \mathrm{mAb}$ binding is the recognition of the GalNAc moiety and to a lesser extent the $\mathrm{Thr}$ residue and the neighbouring downstream proline residue. 5E5 tolerates well any amino acid at APGST*XP, as well as any single mutation in the tandem repeat sequence not located in the epitope and recognizes a conformer low populated in solution. This can be useful in designing novel antigens to formulate new anticancer vaccines and develop novel kits for cancer detections. Funding from the Spanish MCIU (RTI-2018-099592-B-C21, CTQ201344367-C2-2-P, BFU2016-75633-P and PID2019-105451GB-I00), ARAID, the Italian Ministry of Education, University and Research (PRIN 2015 contract nr. 2015RNWJAM), the Royal Society (URF\R\180019) and FCT Portugal (iFCT, IF/00624/2015, and Doctoral Studentship SFRH/BD/111556/2015, IF/00780/2015; PTDC/BIA-MIB/31028/2017) is acknowledged. I.A. B and A. A. thank the $A E C C$ for predoctoral fellowships. E.J.-M. thanks Universidad de La Rioja for a postdoctoral fellowship.

\section{Conflicts of interest}

There are no conflicts to declare.

\section{Notes and references}

1 R. Bhatia, S. K. Gautam, A. Cannon, C. Thompson, B. R. Hall, A. Aithal, K. Banerjee, M. Jain, J. C. Solheim, S. Kumar and S. K. Batra, Cancer Metast. Rev., 2019, 38, 223-236.

2 T. Ju, V. I. Otto and R. D. Cummings, Angew. Chem. Int. Ed., 2011, 50, 1770-1791.

3 C. Fu, H. Zhao, Y. Wang, H. Cai, Y. Xiao, Y. Zeng and H. Chen, $H L A, 2016,88,275-286$.

4 S. K. Kračun, E. Cló, H. Clausen, S. B. Levery, K. J. Jensen and O. Blixt, J. Proteome Res., 2010, 9, 6705-6714.

5 O. Blixt, D. Bueti, B. Burford, D. Allen, S. Julien, M. Hollingsworth, A. Gammerman, I. Fentiman, J. TaylorPapadimitriou and J. M. Burchell, Breast Cancer Res., , DOI:10.1186/bcr2841.

6 M. Movahedin, T. M. Brooks, N. T. Supekar, N. Gokanapudi, G. J. Boons and C. L. Brooks, Glycobiology, 2017, 27, 677687.

7 N. Martínez-Sáez, J. M. Peregrina and F. Corzana, Chem. Soc. Rev., 2017, 46, 7154-7175.

8 I. Compañón, A. Guerreiro, V. Mangini, J. Castro-López, M. Escudero-Casao, A. Avenoza, J. H. Busto, S. Castillón, J. Jiménez-Barbero, J. L. Asensio, G. Jiménez-Osés, O. Boutureira, J. M. Peregrina, R. Hurtado-Guerrero, R. Fiammengo, G. J. L. Bernardes and F. Corzana, J. Am. Chem. Soc., 2019, 141, 4063-4072.

9 U. Karsten, N. Serttas, H. Paulsen, A. Danielczyk and S. Goletz, Glycobiology, 2004, 14, 681-692.

10 Y. Yoshimura, K. Denda-Nagai, Y. Takahashi, I. Nagashima, H. Shimizu, T. Kishimoto, M. Noji, S. Shichino, Y. Chiba and T. Irimura, Sci. Rep., 2019, 9, 16641.

11 M. A. Tarp, A. L. Sørensen, U. Mandel, H. Paulsen, J. Burchell, J. Taylor-Papadimitriou and H. Clausen, Glycobiology, 2007,
17, 197-209.

View Article Online

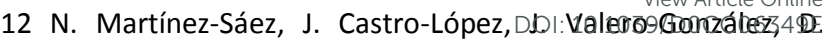
Madariaga, I. Compañón, V. J. Somovilla, M. Salvadó, J. L. Asensio, J. Jiménez-Barbero, A. Avenoza, J. H. Busto, G. J. L. Bernardes, J. M. Peregrina, R. Hurtado-Guerrero and F. Corzana, Angew. Chem. Int. Ed. Engl., 2015, 54, 9830-9834.

13 C. L. Brooks, A. Schietinger, S. N. Borisova, P. Kufer, M. Okon, T. Hirama, C. R. MacKenzie, L. X. Wang, H. Schreiber and S. V. Evansa, Proc. Natl. Acad. Sci. USA, 2010, 107, 10056-10061.

14 H. Wakui, Y. Tanaka, T. Ose, I. Matsumoto, K. Kato, Y. Min, T. Tachibana, M. Sato, K. Naruchi, F. G. Martin, H. Hinou and S.I. Nishimura, Chem. Sci., 2020, 11, 4999-5006.

15 A. D. Posey, R. D. Schwab, A. C. Boesteanu, C. Steentoft, U. Mandel, B. Engels, J. D. Stone, T. D. Madsen, K. Schreiber, K. M. Haines, A. P. Cogdill, T. J. Chen, D. Song, J. Scholler, D. M. Kranz, M. D. Feldman, R. Young, B. Keith, H. Schreiber, H. Clausen, L. A. Johnson and C. H. June, Immunity, 2016, 44, 1444-1454.

16 Y. He, K. Schreiber, S. P. Wolf, F. Wen, C. Steentoft, J. Zerweck, M. Steiner, P. Sharma, H. M. Shepard, A. Posey, C. H. June, U. Mandel, H. Clausen, M. Leisegang, S. C. Meredith, D. M. Kranz and $\mathrm{H}$. Schreiber, $\mathrm{JCl}$ Insight, DOI:10.1172/jci.insight.130416.

17 P. Sharma, V. V. V. R. Marada, Q. Cai, M. Kizerwetter, Y. He, S. P. Wolf, K. Schreiber, H. Clausen, H. Schreiber and D. M. Kranz, Proc. Natl. Acad. Sci. USA, 2020, 117, 15148-15159.

18 I. A. Bermejo, I. Usabiaga, I. Compañón, J. Castro-López, A. Insausti, J. A. Fernández, A. Avenoza, J. H. Busto, J. JiménezBarbero, J. L. Asensio, J. M. Peregrina, G. Jiménez-Osés, R. Hurtado-Guerrero, E. J. Cocinero and F. Corzana, J. Am. Chem. Soc., 2018, 140, 9952-9960.

19 F. Corzana, J. H. Busto, G. Jiménez-Osés, M. García de Luis, J. L. Asensio, J. Jiménez-Barbero, J. M. Peregrina and A. Avenoza, J. Am. Chem. Soc., 2007, 129, 9458-9467.

20 O. Blixt, E. Cló, A. S. Nudelman, K. K. Sørensen, T. Clausen, H. H. Wandall, P. O. Livingston, H. Clausen and K. J. Jensen, J. Proteome Res., 2010, 9, 5250-5261.

21 O. Blixt and E. Cló, Methods Mol. Biol., 2013, 1047, 201-214.

22 F. Corzana, J. H. Busto, G. Jiménez-Osés, J. L. Asensio, J. Jiménez-Barbero, J. M. Peregrina and A. Avenoza, J. Am. Chem. Soc., 2006, 128, 14640-14648.

23 D. Madariaga, N. Martinez-Sáez, V. J. Somovilla, H. Coelho, J. Valero-González, J. Castro-Lopez, J. L. Asensio, J. JiménezBarbero, J. H. Busto, A. Avenoza, F. Marcelo, R. HurtadoGuerrero, F. Corzana and J. M. Peregrina, ACS Chem. Biol., 2015, 10, 747-756.

24 L. Kinarsky, G. Suryanarayanan, O. Prakash, H. Paulsen, H. Clausen, F. G. Hanisch, M. A. Hollingsworth and S. Sherman, Glycobiology, 2003, 13, 929-939.

Author Contributions. I.A.B., A.A., I.C., V.M. and R.F. synthesized, purified and characterized the glycopeptides. F.C. performed and analyzed the MD simulations. J.M.-L., I.S.A., G.J.L.B. and R.H.-G. purified antibody scFv-5E5. J.M.-L. and R.H.G. crystallized the complex and refined the crystal structure. R.H.-G. and A.G.-G. performed the ITC and microscale thermophoresis experiments. O. B. and H.J.J. accomplished the microarray assays. H.C., F.M., J.L.A, E.J-M. and F.C. performed the conformational analysis of glycopeptide MUC1-Tn16 by combining NMR and MD simulations. H.C. and F.M. performed the STD-NMR experiments. F.C. and R.H.-G. wrote the article with the other authors' contributions. 
The molecular basis of an anti-MUC1 antibody that recognizes the entire GalNAc unitView Article Online as a primary epitope is disclosed.

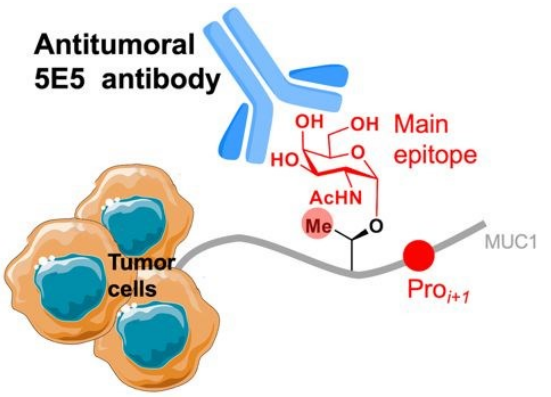

